\title{
Peligros del Tecno-optimismo
}

\author{
doi: 10.52749/fh.v2i1.10
}

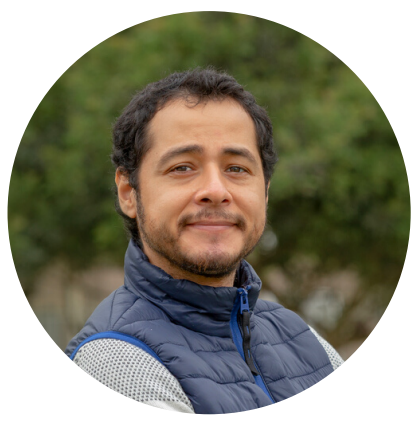

\section{RAÚL QUIROZ OLASCOAGA}

Licenciado en Arte y Diseño Gráfico por el Instituto San Ignacio de Loyola. Apasionado por el estudio de las nuevas tecnologías. Miembro e investigador de la Sociedad Secular Humanista del Perú y del Instituto de Extrapolítica y Transhumanismo.

holaraulquiroz@gmail.com

@raulquirozo

Podemos decir que desde que las revoluciones industriales ocurrieron, las sociedades, en su mayoría occidentales, fueron las que ganaron la lotería económica, pues la calidad de vida de estos países aumentó de manera considerable. Pero por efecto rebote la discrepancia y envidia entre naciones se evidenciaron. Solemos creer que son solo las ideologías las que generan polarización, pero vivir en un mundo de contrastes y desigualdades aumenta el recelo y los discursos de odio. Desafortunadamente, la tecnología está sirviendo como megáfono para dividir al mundo y acentuar aún más esas tensiones geopolíticas y sociales.

En nuestros tiempos, se suele tener una visión optimista de la tecnología, una especie de pensamiento mágico. Pero los problemas que suponen un peligro existencial para nuestra especie son en primer lugar más de corte social, humanitario y político. Según el informe de riesgos globales del Foro Económico Mundial, los riesgos globales medidos por impacto, como los desastres naturales, la propagación de enfermedades contagiosas, las crisis hídricas, la escasez de alimentos y los eventos meteorológicos extremos, hacen que nos resulte tentador verlos con un enfoque técnico, pero eso es abrazar un pensamiento religioso, encomendarnos por fe en el método y en la técnica.

¿Cómo podemos reducir la polarización del mundo o la creciente ola nacionalista solo con técnica? Si pensamos de esta manera, siempre abordaremos los problemas desde un enfoque insípido. Nos hemos olvidado de que el método nos ayuda a comprender y contrastar la realidad, de que la técnica nos permite desarrollar y usar herramientas adecuadas y de que cada uno de los problemas del ámbito humano son justamente de competencia humana. Es decir, propias de todas las dimensiones del ser humano, tales como la educativa, la ética y la política.

Pongamos como ejemplo las armas de destrucción masiva. Estas suponen un grave riesgo para la vida en el planeta. Si en una audiencia preguntamos ¿cómo podemos resolver el problema de la proliferación de armamento nuclear? Nunca faltarán quienes digan que la solución es desarrollar sistemas antimisiles de última generación. Esa manera de enfocar los problemas es vacía y da la bienvenida a una creciente ola tecno-optimista que se traduce en una visión absolutamente sesgada de la condición humana.

Si preguntamos cómo podemos suprimir las noticias falsas relacionadas a la salud, nuevamente se levantarán algunos y dirán que los algoritmos de inteligencia artificial son la solución definitiva. A eso llamo visión tecno-sesgada, porque sabemos que tras las noticias falsas hay seres humanos que crean información o algoritmos con contenidos falsos.

Pero no solo hay fanáticos de la tecnología, los hay también del dinero y de los mercados. $Y$ casi siempre se han puesto a estos factores por encima de las personas, y esto ocasiona una profunda crisis humanitaria. En palabras de Nick Bostrom "los seres humanos somos como unos chimpancés manejando un Ferrari", claramente el Ferrari lo puedes reemplazar por tecnología, dinero, poder, etc.

La ciencia y la técnica están siendo nuestras salvadoras en un contexto pandémico en el que no hay vuelta atrás. No podemos regresar al pasado y tomar acciones políticas para prevenir la aparición de la COVID-19. Y como medida de urgencia recurrimos a estas para rescatarnos, pero estamos dejando de lado lo que para mí es el verdadero potencial de la ciencia: la prevención de efectos de- 
safortunados. Por eso creo que, para abordar los problemas sociales, de salud, de contaminación y humanitarios, es necesario utilizar múltiples competencias. Si solo dejamos a la técnica o al método actuar, solo vamos a recurrir a ellas cuando las cosas estén mal.

Hay un debate muy candente en relación al potencial que tiene la ciencia y la tecnología sobre las limitaciones de los seres humanos. Las discusiones sobre la inmortalidad biológica, la creación de la consciencia computacional o la descarga de nuestra mente a una computadora, son cada vez más recurrentes. Claramente son discusiones válidas que nos ayudan a proyectarnos hacia el futuro, pero con respecto a la ciencia y su método, vale analizar si estas son lo suficientemente capaces de explicar con precisión algunos vacíos teóricos como los misterios de la mecánica cuántica para desarrollar seres artificiales conscientes. Diferentes posturas, como la de Kip Thorne y Roger Penrose, sostienen que reducir la consciencia a procesos computacionales es una manera muy poco eficaz de abordar la generación de consciencia y que tal vez se requiera replantear el método científico para que contemple los misteriosos efectos de la mecánica cuántica en la problemática. $Y$ esto pone en contraste algunas posturas tecno-optimistas que dicen que el desarrollo de inteligencias artificiales fuertes serán seres conscientes con percepción de la realidad y capaces de reconocerse en ella, o que en un futuro no muy lejano haremos backup de nuestras mentes en ordenadores (mind uploading).

Las plataformas sociales como Facebook y YouTube están siendo sumamente cuestionadas, el modelo de negocio bajo la cual estas y otras plataformas operan están transformando el comportamiento y transgrediendo la salud mental y privacidad de los usuarios. De hecho, estas plataformas están dispuestas a aplicar cualquier mecanismo algorítmico para ganarse la atención de las personas. Los discursos de odio, la manipulación de datos, las noticias falsas que se generan y difunden en las redes sociales están fragmentando las sociedades del mundo.

Pero es ahí adonde me dirijo, la gran mayoría de las personas creemos que la violencia no se soluciona con más violencia, al igual que la pobreza no se soluciona con bonos, tampoco hay motivos para pensar que las desigualdades o conflictos se deban solucionar con tecnología. La tecnología es y será un recurso valioso para resolver problemas, pero los problemas sociales complejos que se originan por las nuevas tecnologías de la información se deben detectar, reflexionar, analizar y planificar con la presencia del factor humano, y aunque en cada uno de estos pasos debe estar presente la técnica, hay una gran relevancia de la ética, de la filosofía y de la política.

La evolución tecnológica cada vez se acelera a un ritmo que supone un reto a nivel de gestión política y comunitaria. Siempre la tecnología ha excedido nuestra capacidad de asumir con responsabilidad sus efectos, y eso no es algo nuevo. Cada gran innovación irrumpió de manera abrupta en todos los ámbitos humanos, y si la tecnología tiene esa capacidad, puede que llegue un momento en donde las disrupciones tecnológicas supongan graves peligros existenciales. ¿Puede hacerse?, esta es la pregunta que resuena con más fuerza en la sociedad, pero si algo puede hacerse ¿necesariamente debe hacerse? En un futuro cercano muchas cosas que creíamos ciencia ficción podrán hacerse, pero es ahí donde aparece la ética para cuestionar sus implicancias en la sociedad, y la política para planificar y ejecutar esos beneficios que la tecnología nos brinde.

Como humanidad es hora de dejar de ser infantiles, reemplazar un dogma por otro es una manera muy inmadura de convivir. Reemplazar a la religión por la tecnología solo nos llevará al caos. Lo que necesitamos es asumir responsabilidad y hacer un llamado a actuar con mesura y prudencia.

\section{Bibliografía}

Peirano, M. (2019). El enemigo conoce al sistema. Debate Penrose, R. (1994). Las sombras de la mente. Crítica

Dieguez, A. (2017). Transhumanismo. Herder
Schwab, K. (2016). Cuarta Revolución Industrial. Debate Harari, Y. (2018). 21 lecciones para el siglo XXI. Debate

\section{Cómo citar este artículo:}

Quiroz Olascoaga, R. (2021). Peligros del Tecno-optimismo. Futuro Hoy, 2(1), 35-36. https://doi.org/10.5281/zenodo.4654908 